
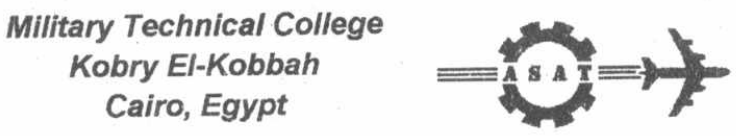

$10^{\text {th }}$ International Conference

On Aerospace Sciences\&

Aviation Technology

\title{
COGENERATION IN INTERNAL COMBUSTION \\ ENGINES (ICE) USING DIRECT BOILING \\ IN THE COOLING JACKETS
}

\author{
Salman E. Elshamarka *
}

\begin{abstract}
Cooling of combustion chamber in an ICE is fulfilled by changing the phase of the cooling medium instead of increasing its temperature. This have been applied considering the thermodynamic properties of the coolant and how much heat can be transferred from engine walls to a second user. The study emphasizes on the concept of cogeneration rather than cooling, waste heat recovery, or enhancing engine performance separately.

Based on cogeneration concepts, design and selection of the cooling system led to generation of additional mechanical power from an organic Rankine cycle (ORC) using R113 as the working medium. At the same time R113 works as the coolant of cylinder walls. Walls, here, represent the furnace in the boiler of the ORC.

However, implementing this method proves the added value of selecting a cooling system that produces a useful output. Results show that the most significant factors affecting the system performance are good match between cooling requirement for the engine and enthalpy addition to the working medium for the ORC, and the influence of heat transfer mechanism from cylinder wall to coolant. Comparison with typical energy and exergy analyses for other power plants showed that merits are anticipated from applying evaporative cooling with ICE's.
\end{abstract}

\section{KEY WORDS}

Internal Combustion Engine, Waste Heat Recovery, Exergy Analysis, Cogeneration.

\footnotetext{
* Egyptian Armed Forces
} 


\section{NOMENCLATURE}

$\begin{array}{ll}E & \text { exergy rate } \\ H & \text { enthalpy } \\ P & \text { power } \\ Q & \text { heat } \\ W & \text { work } \\ \eta & \text { efficiency }\end{array}$

\section{SUBSCRIPT}

$\begin{array}{ll}\text { a } & \text { added } \\ b & \text { brake } \\ c, e & \text { coolant, exhaust } \\ \text { cool } & \text { cooling } \\ \text { eg } & \text { effective from gas turbine } \\ \text { e,ic } & \text { exhaust, incomplet combustion } \\ \text { e, } \mathrm{k} & \text { exhaust, kinetic energy } \\ e, r & \text { exhaust, radiation } \\ \text { e,s,a } & \text { exhaust, sensible, atmosphere } \\ \text { es } & \text { effective from steam turbine } \\ \text { exh } & \text { exhaust } \\ f & \text { fuel } \\ \text { i } & \text { indicated } \\ \text { LHV } & \text { lower heating value } \\ \text { misc } & \text { miscellaneous } \\ p & \text { pump } \\ \text { pf } & \text { friction parts } \\ Q_{H} & \text { heat added } \\ \text { rad } & \text { radiation } \\ \text { rej } & \text { rejection } \\ t & \text { turbine } \\ w & \text { water } \\ x & \text { flowing stream } \\ & \end{array}$

\section{I- INTRODUCTION:}

Internal Combustion Engine is widely used as a power generator, either for motorized vehicles, or for electric power applications. Range of power produced from such engines includes fraction of kW and goes up to hundreds of kW's. This makes ICE a popular power arrangement compared with gas turbine or steam power plants.

However, efficiency and quality of energy dissipated from ICE's represent an important drawback which challenged researchers towards enhancing combustion process, fuel feeding and preparation systems, and other supporting mechanisms. 
References show very great number of contributions in solving such problems, developing ICE performance, enhancing cooling effects, and treating gases to abide with environmental regulations. In some cases researches did many successful proposals for waste heat recovery.

Combined cycle solved some problems in both gas turbine and steam power plants, as well as increasing the overall efficiency by merging them together in one plant. Now such plants are in service and are appreciated by industry. Similar thinking has not been devoted to solve problems of ICE.

It is well known that one fourth to one third of input heat is dissipated by cooling system of ICE, [1]. Instead of tracing this quantity of heat or trying to minimize it (adiabatic engine) in one hand, or decreasing its quality in another hand, a different solution is proposed in this study.

Applying cogeneration concept while designing ICE's, initiates ideas for benefiting from the output energy streams of the engine, mechanical shaft work, heat in cooling system, and heat in exhaust system. This way of thinking way, in some cases, requires increasing cooling heat rather than decreasing it, and generally is looking at what was thought as a drawback as a potential gain, for further utilization.

It was proposed, here, to apply evaporative cooling of ICE combustion chamber walls in order to capture this heat to be used for another power cycle producing addition power such that total system efficiency is increased.

Typical energy flow diagrams (or energy audit) for ICE, gas turbine, steam power plants and combined cycles are reviewed. The proposed new plant energy flow diagram is estimated. A proposed cycle arrangement is elaborated.

It is recommended to apply such arrangement which reduces efforts and costs of implementing a cooling system to an ICE, while adding considerable power to the total product and open new area for applying organic Rankine cycles.

\section{II-PROPOSED ARRANGEMENT:}

The proposed system considers the ICE cylinder walls as the heating surface of the Rankine cycle boiler (evaporator). Heat is transferred from the metallic walls (may be finned) to the working medium (coolant, or refrigerant) in the cooling jacket.

Evaporated working medium may be superheated by adjusting the period of stay in the jacket (mass flow control). It is then allowed to expand in an expander (lowgrade energy one), where mechanical power is produced. Output from the expander is passed into water cooled condenser and so the cycle continues through receiver (or reservoir), preheaters ( if required by designer), and feed pumps.

Produced shaft power may be used directly to drive engine accessories, to power take-off's, to drive electric power generators, or be added to ICE output through efficient mechanical arrangement. 


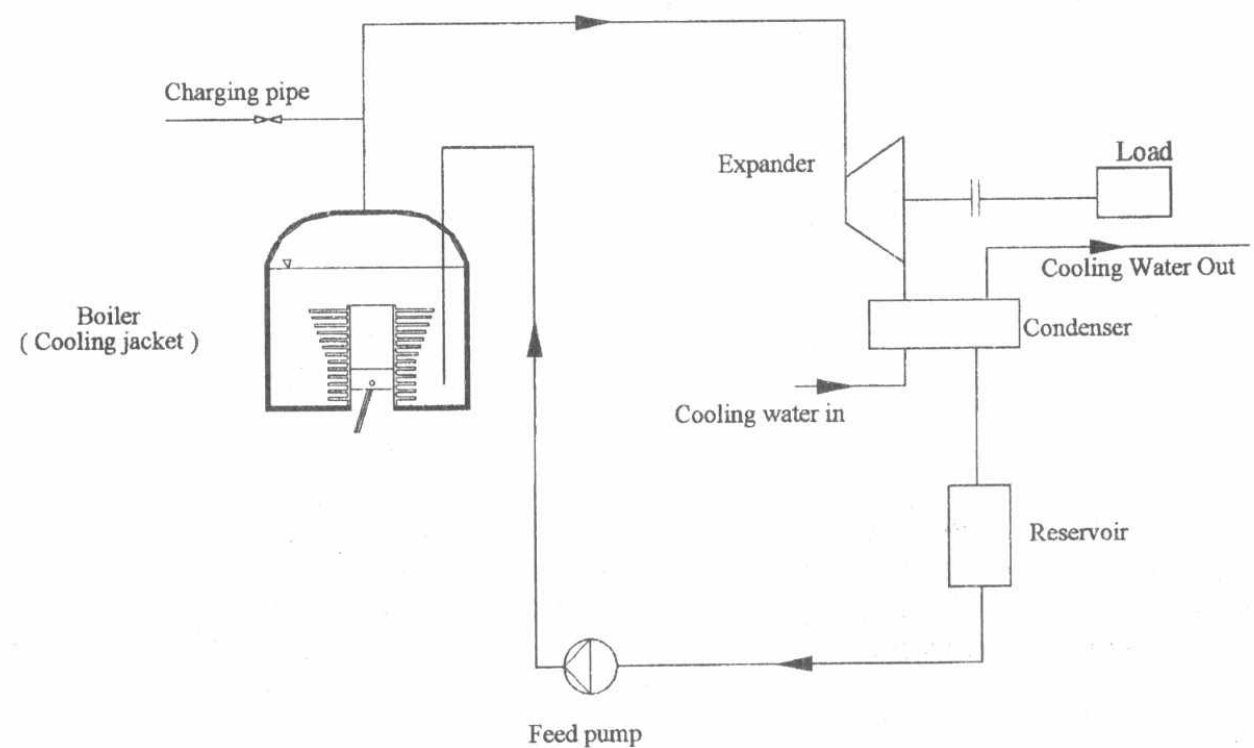

Fig. 1 Schematic drawing of a proposed evaporative cooling system of ICE

\section{II.1 Working medium:}

Selecting the working medium is constrained by the following:

1- the designed Rankine cycle is bottomed by the heat source (at ICE wall temperatures).

2- moderate pressures in the jacket are favorable (strength \& size constraints).

3- required cooling rate of ICE.

4- thermal and chemical properties have to be convenient for pressures, temperatures, and mass flow rates, in addition to component preferences encountered with such low-grade energy Rankine cycles.

Generally, organic fluids, rather than water, are preferred and expected to fulfill the above requirements. Wide series of refrigerant may be considered for given design parameters, [2]. 


\subsection{Important Notes:}

The significance of the system considered is the abandonment of the heat transfer equipment between the ICE cooling system and the Rankine cycle evaporator. This would account for less heat exchange penalty, hence better effectiveness, and so better Second-Law efficiency of the system.

Temperature at which heat is transferred from ICE to Rankine cycle is to be decided through optimization techniques for maximum power output or for maximum SecondLaw efficiency subjected to mechanical and environmental constrained.

\section{III-ENERGY FLOW DIAGRAMS:}

Energy audit of the proposed system may result in energy flow diagram (sanky diagram) that clarifies the expected benefits. Fig. 2 represents energy flow of a typical steam power cycle with reheat as well as regenerative modifications. Output work $W$ is about $35 \%$ of input heat to steam generator $\left(Q_{a 1}+Q_{a 2}\right)$.

When combined with a gas turbine cycle, the total output $W_{\text {es }}+W_{\text {eg }}$ is close to $50 \%$ of input heat to the gas turbine - steam turbine cycle steam generator $\left(Q_{a 1}+Q_{a 2}\right)$, Fig.3.

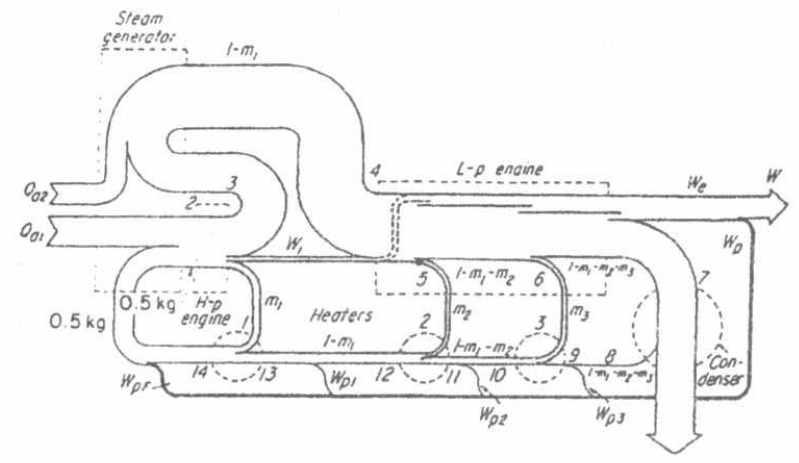

Fig. 2 Energy flow diagram for a typical Steam turbine cycle, with reheat and regeneration, Ref. [3] 


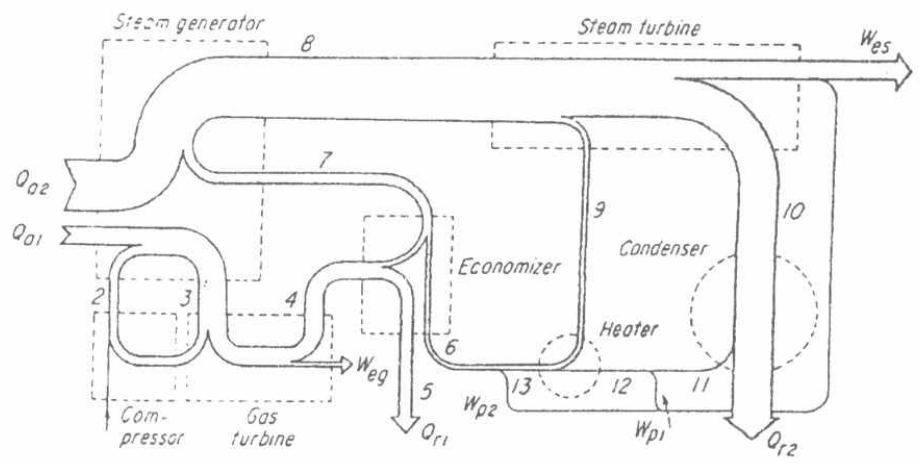

Fig. 3 Energy flow diagram for a typical Gas turbine - steam turbine cycle, Ref. [3]

Fig. 4 shows a typical energy flow diagram for an internal combustion engine, where output power represents about $35 \%$ of input heat rate released from burning of fuel mass flow rate of $m_{f}$ having lower heating value $Q_{\text {LHV }}$. Collected heat rate from hot walls adds to $Q_{w l}$ which is estimated by one fourth to one third of input rate, depending on engine loading.

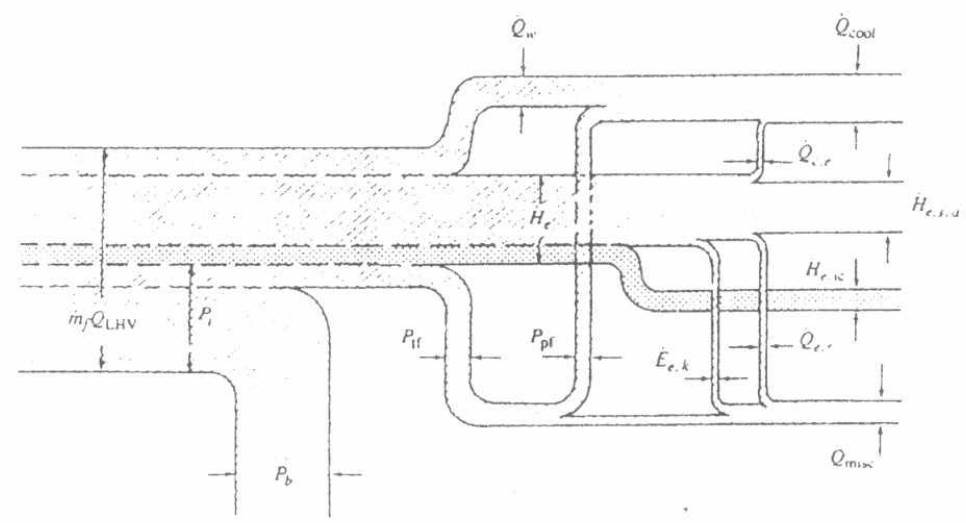

Fig. 4 Energy flow diagram for a typical ICE, Ref. [1] 


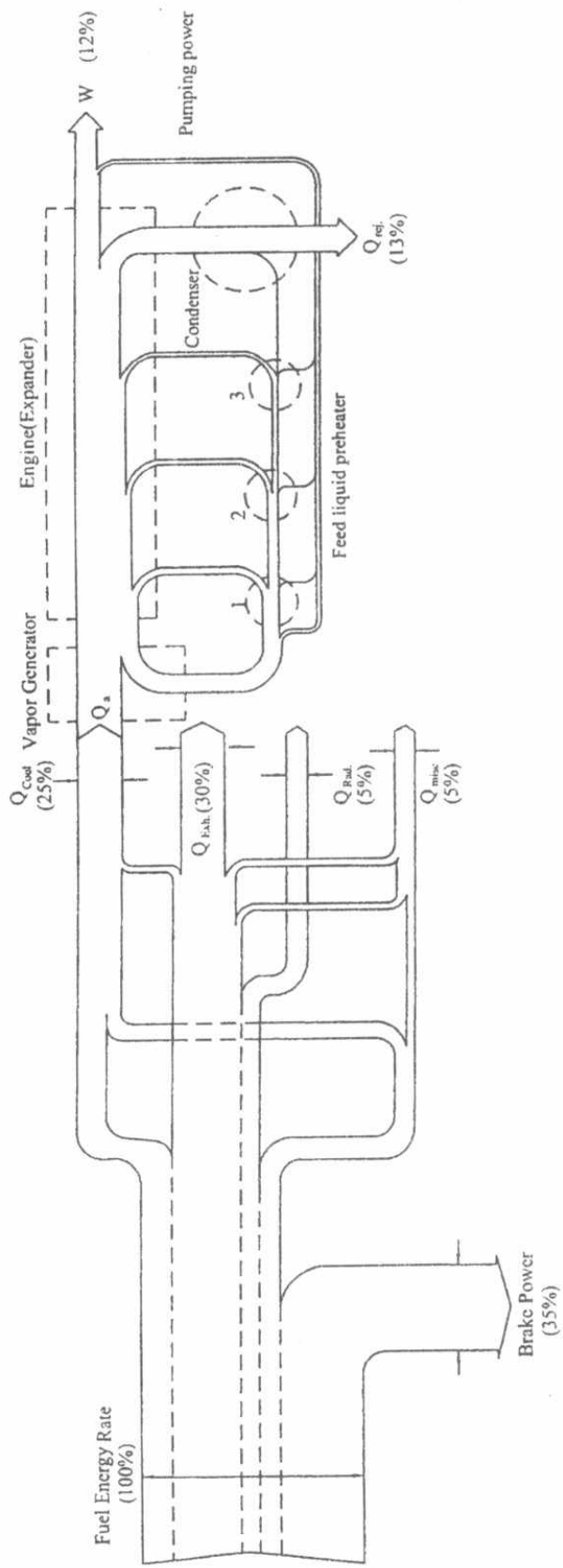

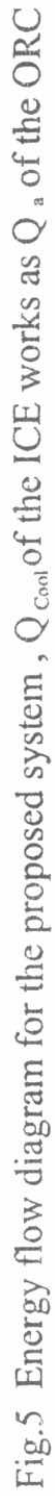




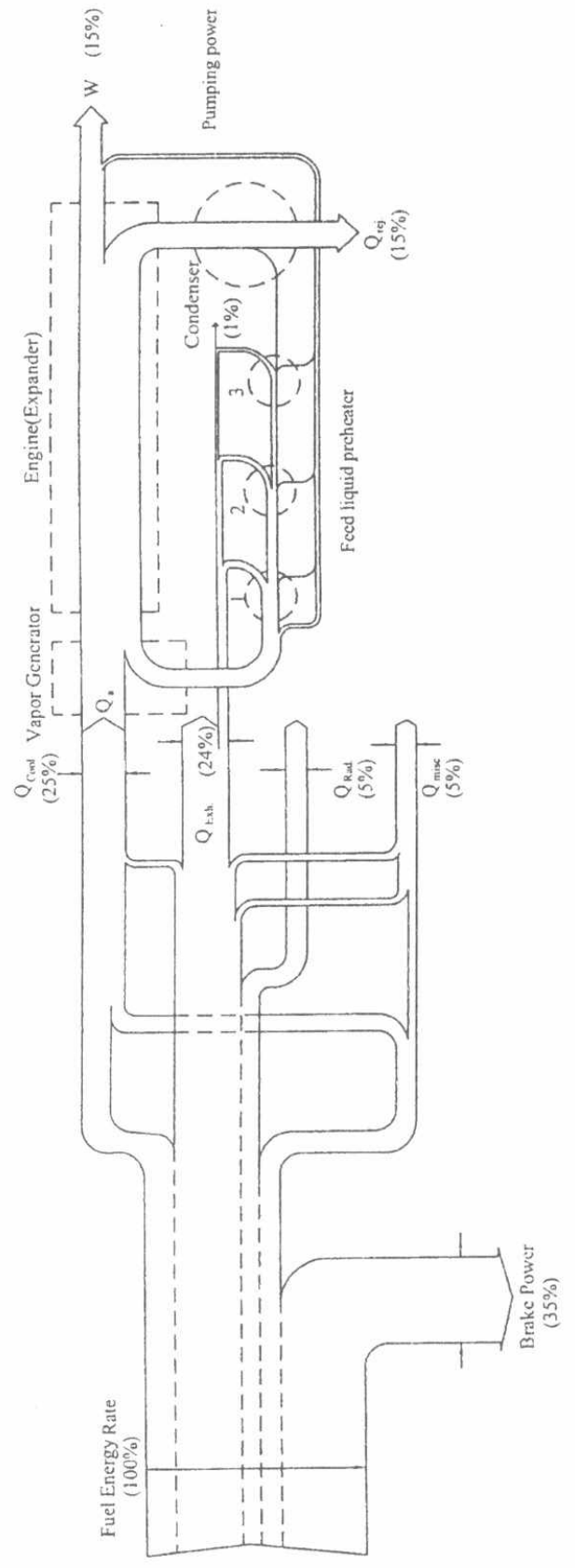

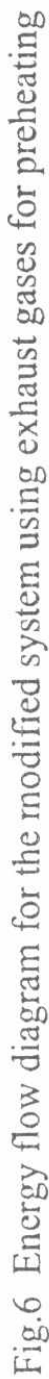


Considering this quantity of heat rate as an input for an organic Rankine cycle (ORC) \& using typical estimates for similar plants working with low-grade energies, an energy flow diagram for the proposed cogeneration system may be shown as that of Fig. 5.

From the figure, it is predicted that additionally produced power $W$ is about $30 \%$ of engine brake power Improvements may be done by preheating feed liquid, in closed heaters, by flue gases enthalpy rather than extracted vapour which certainly increases produced power.

In such system a total efficiency of $45 \div 50 \%$ is expected.

It is worthy to discuss advantages and disadvantages of applying cogeneration in industry. Chiogioji, [4] collected studies about applied plants, mainly using either combined cycles or producing process heat in addition to electricity. In his work, he reported some technical and economical barriers to cogeneration. However, industry as well as governments are willing to apply the concept for its final fuel savings and economic and environmental impacts. Meanwhile, the proposed concept of this work (evaporative cooling of ICE), solves most of the engine problems, while adding excess product.

\section{III-1 Exergy flow diagrams and second-low efficiency:}

When exergy, rather than energy, is traced through the system, exergy flow diagrams can be drawn. They better evaluate the system performance, since they point out the useful part of energy \& picks up sources of exergy destruction. Fig.7 shows a typical one for a Rankine cycle, [5]. The term $\eta_{\|}$stands for the Second-Law efficiency, which figures out how much output exergy obtained from a given input exergy. It is defined for any thermodynamic system.

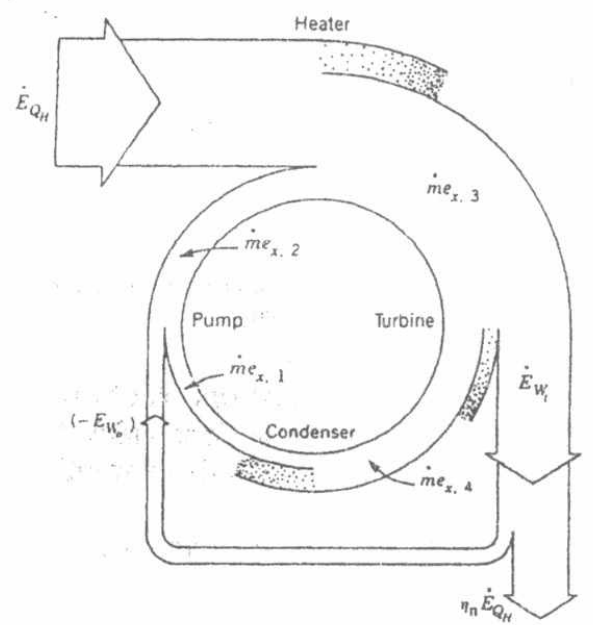

Fig. 7 Exergy flow diagram of a typical Rankine cycle, Ref. [5] 
Similar analysis is made by Haywood, [1] for a typical ICE, for a naturally aspirated and a turbocharged cases. It is evident that, from table 1 that $21.4 \%$ \& $17.6 \%$ of input exergy, is destroyed with heat transferred to cooling system. Turbocharging saved $6 \%$ of input exergy from exhaust gases, and so increasing the engine output by $5 \%$.

Dealing with exergy in the cooling system through the proposed system may save about $10 \%$ of input exergy to the engine (a typical $\eta_{\|}=50 \%$ is common with Rankine cycles). Then a Second-Law efficiency of $50 \%$ for the system is strongly anticipated.

Table 1 Comparison of First and Second Law analyses for a typical ICE, Ref. [1]

\begin{tabular}{|l|c|c|c|}
\hline \multirow{2}{*}{} & \multicolumn{2}{|c|}{ Naturally aspirated } & Turbocharged \\
\cline { 2 - 4 } & $\begin{array}{c}\text { First Law, } \\
\text { \% fuel } \\
\text { energy }\end{array}$ & \multicolumn{2}{|c|}{$\begin{array}{c}\text { Second Law, } \\
\text { \% fuel } \\
\text { availability }\end{array}$} \\
\hline Indicated work & 40.3 & 39.1 & 43.9 \\
Combustion loss & - & 15.9 & 19.2 \\
Cylinder heat transfer & 25.1 & 21.4 & 17.6 \\
Internal valve throttling & - & 0.7 & 0.7 \\
Exhaust valve throttling & - & 2.5 & 2.3 \\
Loss in compressor & - & - & 1.4 \\
Loss in turbine & - & - & 14.1 \\
Exhaust to ambient & $\underline{34.6}$ & 100.4 & 100.0 \\
Total & 100.0 & & \\
\hline
\end{tabular}

\section{IV-CONCLUSION:}

The proposed arrangement, using cogeneration concept satisfies the following:

1- Generating shaft power from ICE at a higher efficiency than typical values, (about 10\% increase).

2- Saving exegy lost in cooling heat. Exergy in the cooling heat is typical a reasonable part of the input exergy.

3- Offering a more effective alternative for the classical cooling system.

\section{REFERENCES:}

1. Heywood, J.B., "Internal Combustion Engine Fundamentals", McGraw Hill, Inc Publishers, New York, USA, 1988.

2. ASHRAE, Fundamentals, 1997

3. Hicks, T.G., Editor, "Standard Handbook of Engineering Calculations, $2^{\text {nd }}$ ed.", McGraw Hill Inc Publishers, New York, USA, 1985.

4. Chiogioji, H., M., "Industrial Energy Conservation", Marcel Dekker, Inc, Publishers, New York, USA, 1979.

5. Bejan, A., "Advanced Engineering Thermodynamics", John Wiley \& Sons Publishers, New York, USA, 1988. 